\title{
Polyphenols with indirect proprotein convertase inhibitory activity
}

\author{
JINGJING ZHU ${ }^{1}$, WIM VAN DE VEN ${ }^{1,2}$ and ALPHONS VERMORKEN ${ }^{1}$ \\ ${ }^{1}$ Laboratory of Molecular Oncology, Department of Human Genetics, KU Leuven, B-3000 Leuven, Belgium; \\ ${ }^{2}$ Charles E. Schmidt College of Science, Florida Atlantic University, Boca Raton, FL 33431, USA
}

Received April 17,2013; Accepted May 30, 2013

DOI: 10.3892/ijo.2013.2009

\begin{abstract}
Polyphenols, a class of natural products, have been shown to exhibit cancer protective properties. Proprotein convertases form a family of mammalian subtilisin-like serine endoproteases. Increased expression of these enzymes has been associated with numerous pathologies including cancer. It has been suggested that the cancer protective effect of polyphenols might be related to their proprotein convertase inhibitory effects. Furin, the most studied proprotein convertase, was shown to be inhibited by polyphenols in an in vitro fluorescence peptidebased assay. Protein substrates or the presence of protein prevented this inhibition by prototype members of various classes of polyphenolic compounds. Inhibition appeared to be related to the reactivity of polyphenol auto-oxidation products to proteins. While direct inhibition by polyphenols of furin has, therefore, not been observed in cells, the existence of indirect mechanisms cannot be excluded. In the present investigation, 26 polyphenols and 5 control compounds were screened for indirect inhibition of furin in a cellular environment. Five polyphenols showed moderate inhibitory activity and three of these: octyl gallate, dodecyl gallate and nordihydroguariaretic acid were further studied. The processing in cells of several genuine furin substrates, including pro-IGF-1R, appeared to be inhibited by these polyphenols. The inhibition was not specific for furin but also affected other proprotein convertases. The three poly-
\end{abstract}

Correspondence to: Professor Alphons J.M. Vermorken, Laboratory of Molecular Oncology, Department of Human Genetics, KU Leuven, Herestraat 49, bus 602, B-3000 Leuven, Belgium

E-mail: fons.vermorken@med.kuleuven.be

Abbreviations: AMC, 7-amino-4-methyl coumarin; ATCC, American Type Culture Collection; CCL, culture clone; $\mathrm{CHO}$, Chinese hamster ovary cells; D9R, nona-D-arginine; DMEM/F12, Dulbecco's modified Eagle's medium/nutrient mixture F12; DMSO, dimethylsulfoxide; DG, dodecyl gallate; GPC3, glypican 3; IGF-1R, insulin-like growth factor 1 receptor; IGF-1, insulin-like growth factor 1; NA, nordihydroguariaretic acid; OG, octyl gallate; PAGE, polyacrylamide gel electrophoresis; $\mathrm{PC}$, proprotein convertase; $\mathrm{Pyr}$, pyroglutamic acid; TPCK, tosyl phenylalanyl chloromethyl ketone

Key words: polyphenols, proprotein convertases, furin, tumor cell growth, IGF-1R, focus formation, anchorage-independent growth, octyl gallate, dodecyl gallate, nordihydroguariaretic acid phenols inhibited the maturation of the furin zymogen, thereby limiting the formation of the active enzyme. The three polyphenols inhibited focus formation of HepG2 liver carcinoma cells suggesting reversal of the malignant phenotype. Anchorageindependent growth of these cells, a hallmark feature of tumor cells, was also inhibited. Since, dependent of the molecular subclass of hepatocellular carcinoma, overexpression of furin can have either favourable or detrimental effects, it seems advisable to take indirect proprotein convertase inhibitory activity into account when polyphenols are considered for therapy of hepatocellular carcinoma.

\section{Introduction}

Natural products and their derivatives have been demonstrated to be effective in cancer prevention and many active phytochemicals are in human clinical trials (1). Nature indeed provides a high number of compounds for pharmaceutical applications or lead compounds of interest for the design of new anticancer drugs. Their great diversity may reflect the many different biological targets that are critical in cancer-related processes. In this context, polyphenols constitute an extensively studied class of plant-derived compounds with importance as cancer therapeutics (2). Mechanistic aspects of chemoprevention include impact on cell differentiation and cellular growth, effects on activation or inactivation of enzymatic processes, interference with apoptotic processes, inhibition of mutagenesis and metastasis, and also antioxidant action (2). Details about the underlying molecular mechanisms according to which polyphenolic compounds interfere with such cellular processes and exert their therapeutic anticancer capabilities remain often to be determined.

As an example, it has been claimed that several polyphenolic compounds isolated from Oroxylum Indicum displayed in vitro inhibitory activities towards proprotein convertases (PCs). Based on these observations, it was suggested that the inhibitory activities of these polyphenols towards PCs might be responsible for the reported anticancer properties of these compounds (3). The mammalian PC family consists of seven subtilisin-like, $\mathrm{Ca}^{2+}$-dependent serine endoproteases with furin as the prototype (4). PCs convert large inactive precursor proteins into their biologically active mature forms by cleaving peptide bonds at a specific consensus sequence consisting of multiple basic amino acid residues (5). They play a critical role in a wide variety of normal physiological processes and are also implicated in a variety of pathological conditions 
including neurodegenerative diseases and cancer (6-8). As a consequence they are considered therapeutic targets (9). In independent in vitro furin-mediated cleavage studies using a fluorogenic peptide substrate (10), the above-cited inhibitory activities of polyphenols towards PCs could be confirmed as these were demonstrated for a wide variety of polyphenols, including flavanols, flavanonols, flavonols, flavanol gallates, (iso)flavones, and phenolic acids and derivatives. In the latter studies, however, it was also revealed that inhibition of furin-mediated processing by the tested polyphenolic compounds was limited to in vitro cleavage of a fluorogenic peptide substrate only. Similar inhibition of furin-mediated processing of genuine proproteins, like cleaved diphtheria toxin, or processing in cultured cells of tumourigenesislinked proproteins, such as pro-insulin-like growth factor-1 receptor (pro-IGF-IR) and pro-glypican-3 (pro-GPC3), could not be demonstrated (10). For some of the tested polyphenols, it was shown that they inhibit furin processing activity by binding to the furin protein and this could be prevented by the addition of other proteins like bovine serum albumin, casein of $\gamma$ globulin (10). Further studies revealed that polyphenols can inhibit furin in vitro as a result of the reactivity of their auto-oxidation products to proteins (11). In light of the above, it is, therefore, not very likely that the reported cancer preventive or chemotherapeutic activities of the polyphenols tested can be attributed to direct inhibition of furin or other PCs.

In the present study, we therefore added a PC inhibition assay, capable of screening compounds for potential indirect PC inhibitory effects. We tested the polyphenols previously tested (10), added some more and used several non-polyphenolic compounds as controls.

None of the compounds tested showed significant direct inhibitory effects when a genuine protein PC substrate was used. Several polyphenols showed, however, different degrees of indirect inhibition. Three of these polyphenols were studied more extensively. Their PC inhibitory activity was studied in vitro and in cell lines. Their anticancer activity was evaluated by measuring the effect on focus formation and on anchorageindependent growth. These tests were performed on liver carcinoma cell lines.

\section{Materials and methods}

Materials and cell lines. A purified, biologically active, soluble, recombinant human furin fragment [from Dr I. Lindberg] prepared was used for in vitro enzyme assays as previously described (10). The polyphenols were obtained from Sigma-Aldrich and a $10 \mathrm{mM}$ stock solution for use in different experiments was made in ethanol, DMSO according to the instructions by the supplier. As fluorogenic peptide substrate Pyr-RTKR-AMC (Pyr, pyroglutamic acid; AMC, 7-amino-4-methylcoumarin) was used (Bachem). Uncleaved pro-diphtheria toxin was obtained from Merck and the proprotein convertase inhibitor nona-D-arginine (D9R) from Pepscan Presto, Lelystad, The Nederlands.

Cell lines including CHO (ATCC, CCL-61), RPE.40 (12) and HepG2 (ATCC, HB-8065) were grown in DMEM/F12 (1:1) $\left(\right.$ Invitrogen ${ }^{\mathrm{TM}}$ ) supplemented with $10 \%$ fetal calf serum (HyClone) and cultured at $37^{\circ} \mathrm{C}$ in a humidified $5 \% \mathrm{CO}_{2}$ atmosphere.
DNA constructs and transfection. To test the effect of polyphenols on the processing of proprotein substrates by endogenous PCs in cells, full length cDNA encoding proIGF-1R, or HA-tagged pro-GPC3, which constitute established PC substrates, was transfected into $\mathrm{CHO}$ cells or cells of the furin-deficient cell line RPE.40. Full length human $I G F-I R$ cDNA $(h I G F-I R)$ [kind gift of Dr R. Kooijman, VUB, Brussels, Belgium; (13)], and full length human GPC3 cDNA [kind gift of Dr G. David; (14)] were used in the pcDNA3 expression vector. In similar co-transfection experiments, the effect of the compounds on processing of the afore-mentioned proproteins by exogenously expressed human furin, was studied in RPE.40 cells. Furthermore, to test the effect of these compounds on the maturation of PCs, expression constructs encoding wild-type human furin [the 4.1 EcoR1 FUR cDNA (4)] in pcDNA3 vector was transfected into $\mathrm{CHO}$ cells. Transfections were performed using Fugene ${ }^{\mathrm{TM}} 6$ Transfection Reagent (Roche) according to the manufacturer's protocol. About $24 \mathrm{~h}$ after transfection, the PC zymogens, the proprotein substrates and their corresponding processing products were studied by western blot analysis using specific antibodies.

Fluorescence substrate based furin activity assay for screening polyphenols. A possible impact of polyphenols on the cleavage of the fluorogenic Pyr-RTKR-AMC peptide substrate by purified hfurin ${ }^{573}$ in vitro was determined as follows. Experiments were performed in 96-well microtiter plates containing each a $100 \mu \mathrm{l}$ reaction mixture. First, $2 \mu \mathrm{l}$ of hfurin ${ }^{573}$ solution $(10 \mathrm{ng} / \mu \mathrm{l})$ was added to $83.8 \mu \mathrm{l}$ of buffer solution [0.5\% Triton X-100, $0.5 \mathrm{mM}$ $\mathrm{CaCl}_{2}$ and $100 \mathrm{mM}$ HEPES (pH 7.0)]. Subsequently, one of the polyphenolic compounds $(10 \mu \mathrm{l})$ was added at a final concentration of 10 or $20 \mu \mathrm{M}$ and pre-incubation was performed at room temperature for $30 \mathrm{~min}$. Then $4.2 \mu \mathrm{l}$ of the fluorogenic peptide substrate Pyr-RTKR-AMC (final concentration $100 \mu \mathrm{M}$ ) was added to each well and incubation at $37^{\circ} \mathrm{C}$ was continued. The various solvents used were brought in HEPES buffer and have been used as corresponding vehicle controls. The FLUOstar Galaxy fluorometer (BMG Lab Technologies) was used to measure the fluorescence intensity at an emission wavelength of $460 \mathrm{~nm}$ at $37^{\circ} \mathrm{C}$ at 5-min intervals for $1 \mathrm{~h}$. An excitation wavelength of $390 \mathrm{~nm}$ was used. The fluorometric output, which was obtained as relative fluorescence units (RFU), was plotted against time and further processed. All fluorescence data are reported as the means of the measurements of three independent experiments, with error bars showing the standard deviations. Reaction rates were measured as RFU/min. The final reaction rate was calculated by subtracting the RFU/min of the vehicle controls as background. The experiments in which only hfurin ${ }^{573}$ and fluorogenic substrate were mixed in the absence of inhibitor were used as the positive controls. The percentage inhibition was calculated according to the formula $\{[(\mathrm{RFU} /$ $\mathrm{min})$ positive control - (RFU/min)inhibitor $] /(\mathrm{RFU} / \mathrm{min})$ positive control\} x $100 \%$.

In vitro processing of uncleaved diphtheria toxin by hfurin ${ }^{573}$. Purified hfurin ${ }^{573}$ was preincubated with $5 \mu \mathrm{M}$ D9R or different concentrations of polyphenols $(10 \mu \mathrm{M}$ or $20 \mu \mathrm{M})$ in HEPES I buffer (100 mM HEPES, 0.5\% Triton X-100, $0.5 \mathrm{mM} \mathrm{CaCl}_{2}$ ) at room temperature for $30 \mathrm{~min}$. After the preincubation, $3 \mu \mathrm{g}$ of the uncleaved diphtheria toxin (final concentration $0.3 \mu \mathrm{g} / \mu \mathrm{l}$ ) 
was added and incubated at $37^{\circ} \mathrm{C}$ for $1 \mathrm{~h}$. The final concentration of hfurin ${ }^{573}$ was $0.03 \mathrm{ng} / \mu \mathrm{l}$. Processing of the uncleaved diphtheria toxin into the A- and B-fragments was evaluated by SDS polyacrylamide gel electrophoresis analysis and proteins were visualized by Coomassie Blue staining (Merck Chemicals).

Determination of endogenous PC activity. The procedure for measuring the endogenous PC activity was as follows: $\mathrm{CHO}$ cells were grown to confluence and treated in each experiment for $24 \mathrm{~h}$ with different concentrations of different compounds, as indicated in the figures. Cells were washed twice with PBS buffer and then lysed in $100 \mu 1$ TNT buffer [10 mM Tris- $\mathrm{HCl}$ (pH 7.2), $0.15 \mathrm{M} \mathrm{NaCl}, 0.1 \%$ Triton $\mathrm{X}-100$, and protease inhibitor mix (1.0 $\mu \mathrm{M}$ E-64, $0.1 \mu \mathrm{M}$ leupeptin, $1.0 \mu \mathrm{M}$ pepstatin, $0.01 \mathrm{mM}$ TPCK)]. The cell lysate was centrifuged in a minifuge at $13,000 \mathrm{rpm}$ for $1 \mathrm{~min}$. The supernatant was transferred to another tube and the protein concentration was measured using a BCA (bicinchonic acid) protein assay kit (Thermo Scientific) according to the manufacturer's protocol. Each sample was adjusted by TNT buffer to achieve the same protein concentration. In order to measure the PC activity, $25 \mu \mathrm{l}$ of cell lysate was added to each well of 96-well microtiter plates, then $20 \mu \mathrm{l}$ HEPES II buffer [250 mM HEPES (pH 7.0), 0.25\% Triton X-100, $12.5 \mathrm{mM} \mathrm{CaCl}_{2}$, and the above-mentioned protease inhibitor mix] was added before the addition of the fluorogenic peptide substrate Pyr-RTKR-AMC (200 $\mu \mathrm{M}$ final concentration) and incubation at $37^{\circ} \mathrm{C}$ was continued. As controls $25 \mu \mathrm{l}$ cell lysate, for each polyphenol concentration, added to $25 \mu \mathrm{l}$ HEPES II buffer (pH 7.0) were used. Fluorescence was measured at $37^{\circ} \mathrm{C}$ at 5-min intervals for $1 \mathrm{~h}$. An excitation wavelength of $390 \mathrm{~nm}$ and an emission wavelength of $460 \mathrm{~nm}$ were used. The relative $\mathrm{PC}$ activity, after incubation for $1 \mathrm{~h}$, was plotted against the concentration of the polyphenols. Determination of PC processing activity in the medium was performed similarly.

Western blot analysis. Cells were lysed about $24 \mathrm{~h}$ after transfection in SDS-PAGE sample buffer (50 mM Tris-HCl, pH 6.8, $10 \%$ glycerol, $2 \%$ SDS and $4 \% \beta$-mercaptoethanol) and after boiling the solution for $10 \mathrm{~min}$, samples were loaded on a $10 \%$ SDS-polyacrylamide gel. After separation on the basis of molecular weight, proteins were transferred electrophoretically onto Protran ${ }^{\circledR}$ nitrocellulose transfer membranes (Whatmann $\mathrm{GmbH}$ ) and subjected to western blot analysis. Expression of the IGF-IR-related proteins was evaluated using a rabbit antihIGF-1R $\alpha$-subunit antibody (SC-712; Santa Cruz). Expression of the HA-tagged GPC3 protein was evaluated with a mouse anti-HA tag monoclonal antibody (monoclonal antibody 16B12, Covance). Expression of the pro- and mature forms of human furin was detected using the monoclonal antibody MON-148 (Enzo Life Sciences), which specifically recognizes the subtilisin-like catalytic domain of furin (15). The identity of pro-furin was confirmed with a home-made rabbit polyclonal anti-serum raised against the pro-domain of human pro-furin (designated hProfurin $\mathrm{Ab}$ ). As a control, the expression level of actin was evaluated using an anti- $\beta$-actin monoclonal antibody from Sigma-Aldrich.

Monitoring the effects of the polyphenols on maturation of the $P C$ zymogen, pro-furin. To investigate the possible influence of the polyphenols on pro-furin maturation, $\mathrm{CHO}$ cells were transfected with an expression construct encoding full length human pro-furin. Twenty-four hours after transfection with the pro-furin encoding DNA, various concentrations of different polyphenols were added to the culture medium. After 12-h incubation, the culture medium was removed and cells were washed twice with DMEM/F12 medium and cultured overnight in serum-free medium containing the different compounds. Subsequently, culture medium was precipitated using 4 volumes of methanol and the presence of FUR gene-encoded proteins in the medium was visualised by SDS-PAGE and western blot analysis. The cells were lysed in $200 \mu 1$ of TNT buffer, as described above. The lysate was used to determine the FUR gene-encoded proteins in the cell lysate by SDS-PAGE and western blot analysis using the antibodies mentioned above.

Cell viability assay. Cell viability upon treatment with different polyphenols was studied using the liver carcinoma cell line HepG2. Cells were detached by trypsinization, seeded at 5,000 cells/48-well microtiter plate and cultured for $24 \mathrm{~h}$. Then they were treated with various concentrations of different polyphenols, and incubated for an additional $48 \mathrm{~h}$. The effect of different polyphenols on cell growth was examined by the 3-(4,5-dimethylthiazol-2-yl)-2,5-diphenyltetrazolium bromide (MTT) cell viability assay. Briefly, $100 \mu \mathrm{l}$ of MTT solution ( $5 \mathrm{mg} / \mathrm{ml}$; Sigma-Aldrich) was added to each well and incubated for $4 \mathrm{~h}$ at $37^{\circ} \mathrm{C}$. The supernatant was aspirated, and the MTT formazan that was formed by metabolically viable cells was dissolved in $150 \mu \mathrm{l}$ of DMSO, and then monitored by a Dynatech MR5000 microplate reader (Dynatech Corp., Burlington, MA) at a wavelength of $590 \mathrm{~nm}$. The data are expressed as the percentage of viable cells as compared to the number of cells in the control (taken as $100 \%$ viability), as determined by the MTT reduction test.

\section{Focus formation assay}

Impact of polyphenols on focus formation of HepG 2 cells. The impact of different polyphenols on focus formation by HepG2 cells was examined. HepG2 cells were grown to confluence in DMEM containing $10 \% \mathrm{FBS}$ in a 6 -well plate at $37^{\circ} \mathrm{C}, 5 \% \mathrm{CO}_{2}$ for $24 \mathrm{~h}$. After washing twice with $10 \mathrm{mM}$ phosphate-buffered saline (PBS, pH 7.4), cells were treated with different concentrations of octyl gallate, dodecyl gallate or nordihydroguariaretic acid. The cells were kept in an incubator at $37^{\circ} \mathrm{C}$ for 21 days and the medium was changed every other day, each time supplemented with the polyphenols. On day 21 , the foci were washed with PBS, fixed with $70 \%$ ethanol and the foci formed were observed using light microscopy. Data are presented as the percentage of the number of foci formed after treatment with the polyphenols as compared to the non-polyphenol treated control group. The focus-forming potential of HepG2 cells after polyphenol treatment was expressed in terms of percentage of control.

Colony formation in soft agar. The impact of the treatment of liver carcinoma HepG2 cells with different polyphenols on colony formation was investigated. Cells $(10,000$ cells/well $)$ were seeded onto 6-well plates in culture medium (supplemented with $50 \mathrm{ng} / \mathrm{ml}$ IGF-1) containing $0.35 \%$ low-melting agarose (FMC Corp., Rockland, ME) over a $0.7 \%$ agarose layer 


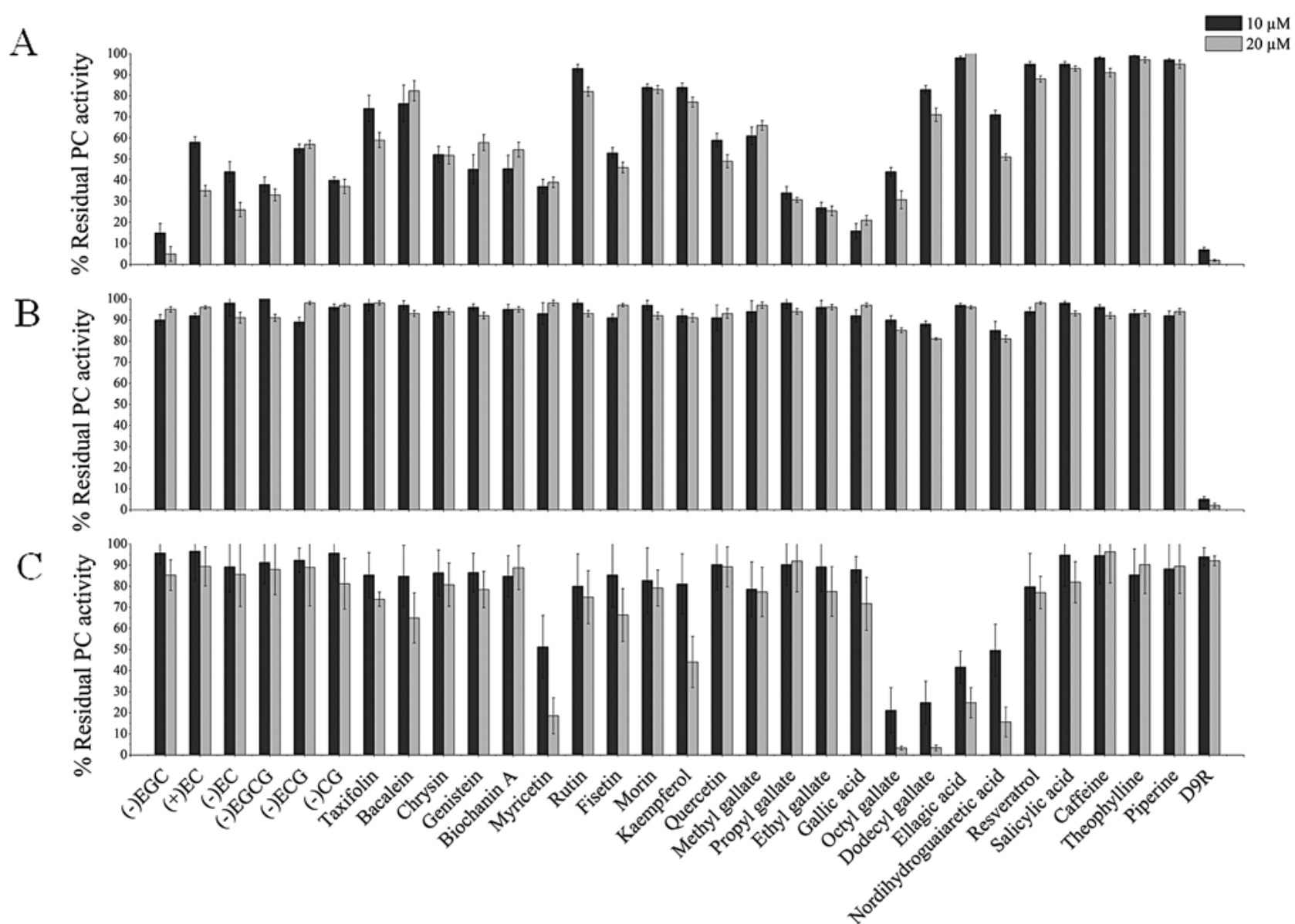

Figure 1. Features of interference by polyphenols and control compounds including D9R with proprotein convertase-mediated processing. (A) Inhibitory effect of the different compounds at the concentrations indicated in the figure on the inhibition of the in vitro hfurin ${ }^{573}$-mediated cleavage of the fluorogenic peptide substrate Pyr-RTKR-AMC. (B) Inhibitory effect of the different compounds at the concentrations indicated in the figure on the hfurin ${ }^{573}$-mediated in vitro processing of uncleaved diphtheria toxin into the A- and B-fragment. (C) Relative endogenous PC activity in CHO cell lysates after treatment of cells with the different compounds at the concentrations indicated in the figure. Treatment of the CHO cells and preparation of the cell lysates were performed as described in Materials and methods. PC-mediated cleavage was assayed in vitro using Pyr-RTKR-AMC. Data represent mean \pm SEM of 3 independent experiments, each performed in triplicate.

in the presence of varying concentrations of polyphenols or control vehicle, as indicated, and incubated for 3 weeks at $37^{\circ} \mathrm{C}$. Fresh medium (supplemented with $30 \mathrm{ng} / \mathrm{ml}$ IGF-1) containing the appropriate concentration of different polyphenols was added and refreshed every other day during the 3-week period. Colony formation in soft agar was monitored using an Axiovert 200M inverted microscope (Zeiss) and scored 3 weeks after seeding of the cells.

Statistical analysis. Data are presented as mean \pm SEM of three independent experiments. Unpaired two-tailed Student's t-test was used for statistical analysis. The graph artwork was created using Microsoft Office Excel 2007 or OriginPro.8.SR0 (OriginLab Corporation, Northampton, MA).

\section{Results}

Comparison of the $P C$ inhibitory effect in different $P C$ activity assays. In vitro fluorescence substrate-based screening assays are commonly used for determining enzyme activity. When the substrate encounters the active enzyme, substrate cleavage will occur and a fluorescence signal will emerge. We have tested 30 compounds. Twenty-six of these are polyphenols, 1 compound is a monophenol (salicylic acid) while 3 compounds are non-polyphenolic small molecules (caffeine, theophylline and piperine). The genuine furin inhibitor D9R was used as a control. These compounds were first tested using the in vitro fluorescence peptide substratebased screening assay and the in vitro assay measuring the processing of uncleaved diphtheria toxin protein.

Fig. 1A shows that the majority of the polyphenols leads to a strong to moderate inhibition of the enzyme activity. Some polyphenols, with ellagic acid as the clearest example, do not provoke inhibition. The genuine PC inhibitor D9R shows a strong inhibitory effect. On the other hand, inhibition by the mono-phenolic and the non-polyphenolic control compounds is weak if at all present.

In light of the previously observed false-positive inhibitory effects of polyphenols on hfurin ${ }^{573}$-mediated cleavage of the fluorogenic peptide substrate in vitro (10), studies were extended to the investigation of the effect of the selected compounds on the in vitro processing of a genuine furin 
proprotein substrate, e.g. uncleaved diphtheria toxin. In contrast to the results obtained with the fluorogenic peptide substrate, no inhibitory effect for any of these 30 compounds was observed on the in vitro processing of the diphtheria toxin proprotein (Fig. 1B). In fact the presence of protein is sufficient to almost completely abolish the inhibitory effects of polyphenols in the observed in vitro fluorescence substratebased screening assay (data not shown). On the contrary, the genuine PC inhibitor D9R strongly inhibits processing also in this assay.

Our results clearly shed doubt on the capacity of the polyphenols tested to inhibit PC activity in vivo, in the presence of abundant other cellular proteins, by direct interaction with the enzyme. On the other hand it cannot be excluded that polyphenols could inhibit PC activity as a result of indirect mechanisms. In order to address this question a cellular test system was used.

The small molecules tested were first incubated with $\mathrm{CHO}$ cells for $24 \mathrm{~h}$. After the incubation, the cells were washed twice with PBS and immediately lysed in TN buffer supplemented with Triton X-100. After lysing the cells, the fluorescent AMC substrate was added and the activity of endogenous PCs was measured. The results indicate that some of the compounds show inhibitory effect in the cell-based assay in which cellular proteins are abundantly present. While a number of compounds show some dose-dependent effect on PC activity (Fig. 1C), only myricitin, octyl gallate, dodecyl gallate, ellagic acid and nordihydroguariaretic acid showed moderate to strong inhibition. Octyl gallate, dodecyl gallate and nordihydroguariaretic acid were used for further studies. It should be noted that D9R has virtually no effect on PC activity in the cellular test system which measures indirect effects on PC activity. This implies that despite the reported capacity of D9R to penetrate cells (16), this is insufficient to reduce $\mathrm{PC}$ activity significantly.

The inhibitory effect of octyl gallate, dodecyl gallate and nordihydroguariaretic acid in $\mathrm{CHO}$ cells is not specific for furin. In order to investigate whether octyl gallate, dodecyl gallate and nordihydroguariaretic acid also inhibit endogenous $\mathrm{PC}$ activity in $\mathrm{CHO}$ cells when other than the fluorescent AMC substrate is used, two genuine PC substrates were tested. The proprotein substrates studied included pro-IGF-1R and pro-GPC3. They were expressed in CHO cell cultures after transfection.

Treatment of the transfected $\mathrm{CHO}$ cells with various concentrations of these three compounds revealed that the ratio between the individual proproteins and their corresponding mature processing products increased at increasing compound concentration (Fig. 2A and B). These results show an inhibitory effect of the three compounds on the processing capabilities of endogenous proprotein convertase in $\mathrm{CHO}$ cells with respect to the three proproteins investigated. The observation that octyl gallate, dodecyl gallate and nordihydroguariaretic acid inhibit endogenous PC-mediated processing of various proproteins raised the question as to whether all $\mathrm{PCs}$ are inhibited by them.

Processing of pro-GPC3 was studied in co-expression experiments using RPE.40 cells. These cells lack furin processing activity as already reported (12). In the presence of various concentrations of octyl gallate, dodecyl gallate
A

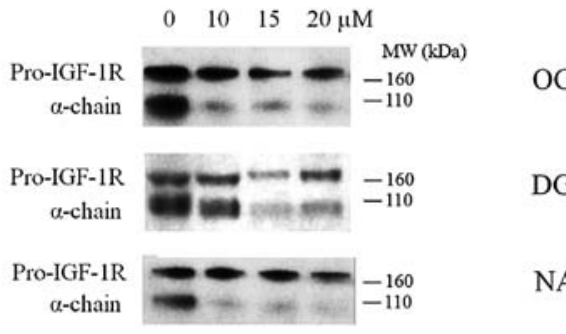

B

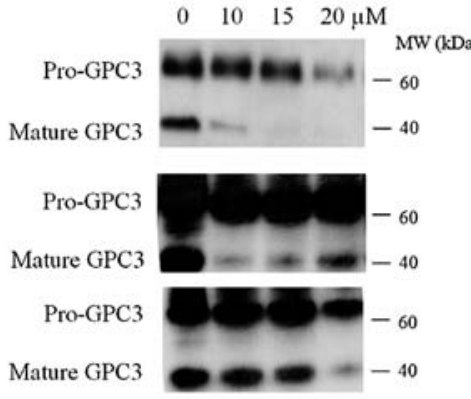

C
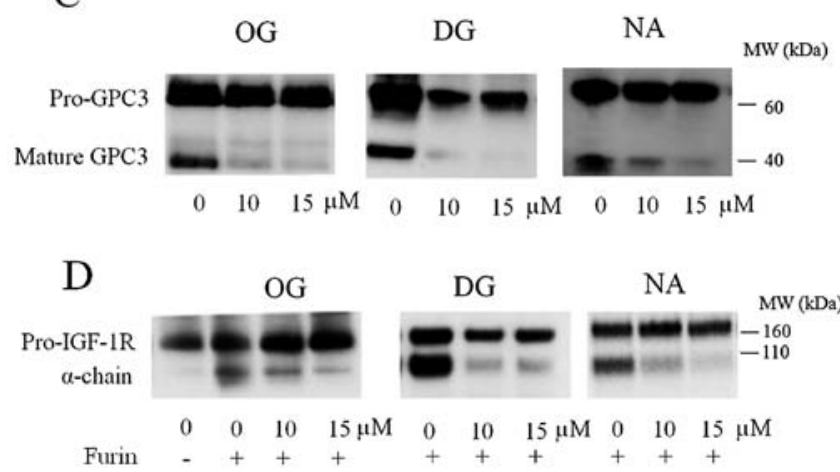

Figure 2. Features of interference by the polyphenols octyl gallate (OG), dodecyl gallate (DG) and nordihydroguariaretic acid (NA) with proprotein convertase-mediated processing of different proproteins in $\mathrm{CHO}$ cells and in RPE.40 cells which lack furin processing activity. Cells were transfected with expression constructs encoding pro-IGF-1R, or pro-GPC 3 and cultured in the presence of various concentrations of the three polyphenols as indicated. (A) Effect of increasing concentrations of the three polyphenols on the ratio between pro-IGF-1R and the corresponding mature processing product the IGF-1R $\alpha$-chain in CHO cells. (B) Effect of increasing concentrations of the three polyphenols on the ratio between pro-GPC3 and the corresponding mature processing product GPC3 in CHO cells. (C) Effect of increasing concentrations of the three polyphenols on the ratio between pro-GPC 3 and the corresponding mature processing product GPC 3 in RPE.40 cells which do not express furin. (D) Effect of increasing concentrations of the three polyphenols on the ratio between pro-IGF-1R and the corresponding mature processing product the IGF-1R $\alpha$-chain in RPE. 40 cells which were or were not co-transfected with furin. Please note that pro-IGF-1R is not processed by the endogenous PCs present in RPE.40 cells (the most left lane in D). The three polyphenols therefore inhibit furin activity.

and nordihydroguariaretic acid, the processing of pro-GPC3 was inhibited (Fig. 2C), suggesting that the endoproteolytic processing activity of endogenous proprotein convertase in RPE.40 cells is affected by these three polyphenols.

This result leaves us with the question whether furin is also inhibited by these three polyphenols. In order to answer this question RPE.40 cells were transfected with constructs 


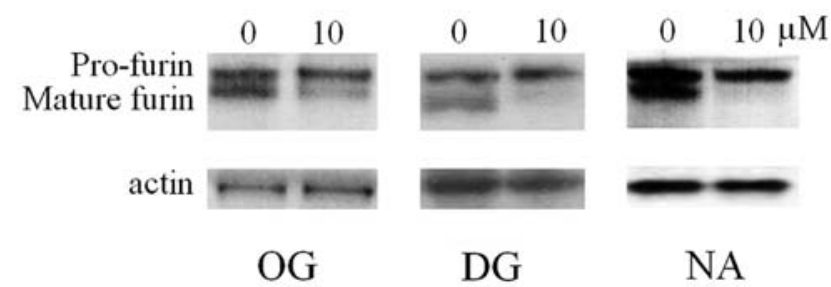

Figure 3. Effect of the polyphenols octyl gallate (OG), dodecyl gallate (DG) and nordihydroguariaretic acid (NA) on zymogen maturation of pro-furin in $\mathrm{CHO}$ cells. Expression levels of actin are shown as protein loading controls. Synthesis of endogenous human pro-furin and production of its mature form furin were studied in $\mathrm{CHO}$ cells by western blot analysis, as described in Materials and methods. In the absence of the three polyphenols pro-furin $(100 \mathrm{kDa})$ and mature furin $(90 \mathrm{kDa})$ could be observed in $\mathrm{CHO}$ cell lysates. In the presence of one of the three polyphenols, as indicated in the figure, the ratio between detectable levels of mature furin and pro-furin decreased, revealing the inhibition of pro-furin maturation.

for furin as well as for the pro-IGF-1R. Pro-IGF-1R appears not to be processed by the endogenous PCs in RPE.40 cells (Fig. 2D). In transfection experiments of RPE.40 cells with constructs for both pro-furin and pro-IGF-1R, octyl gallate, dodecyl gallate and nordihydroguariaretic acid appear capable of inhibiting the processing of pro-IGF-1R (Fig. 2D).

Octyl gallate, dodecyl gallate and nordihydroguariaretic acid inhibit furin maturation in 293 T cells. Proprotein convertase maturation, an intramolecular autocatalytic process, is required for the formation of the active enzyme. Since the inhibitory effect of octyl gallate, dodecyl gallate and nordihydroguariaretic acid on furin activity is not due to the direct inhibition of the enzyme, we next investigated whether the maturation of the enzyme would be influenced by these compounds. Therefore, the maturation of pro-furin was studied by western blot analysis in CHO cells in the presence of $10 \mu \mathrm{M}$ of one of these three inhibitors. At a $10 \mu \mathrm{M}$ concentration of the inhibitors, the amount of pro-furin is increasing and concomitantly, that of intracellular mature furin is decreasing (Fig. 3). The identity of pro-furin was established using rabbit polyclonal antibody hPro-furin $\mathrm{Ab}$ which is specific for the pro-domain of human pro-furin (result not shown).

Octyl gallate, dodecyl gallate and nordihydroguariaretic acid inhibit pro-IGF-1R maturation in HepG2 cells. HepG2 cells form a hepatocellular carcinoma cell line in which the IGF-1R is overexpressed and in which inhibition of IGF-1R signaling with a monoclonal antibody against IGF-1R was shown to reduce cellular proliferation and to induce apoptosis (17). Overexpression of furin promoted HepG2 cell invasion in tail vein xenograft models (18).

In order to investigate whether the indirect PC inhibitory effect of octyl gallate, dodecyl gallate and nordihydroguariaretic acid could potentially be relevant for inhibition of tumor growth, the IGF-1R signaling pathway was studied in the HepG2 hepatocellular carcinoma cell line. HepG2 cells were used to study the inhibitory effect of these compounds on the processing of pro-IGF-1R. After exposure of HepG2 cells to the polyphenols a strong reduction of processing was

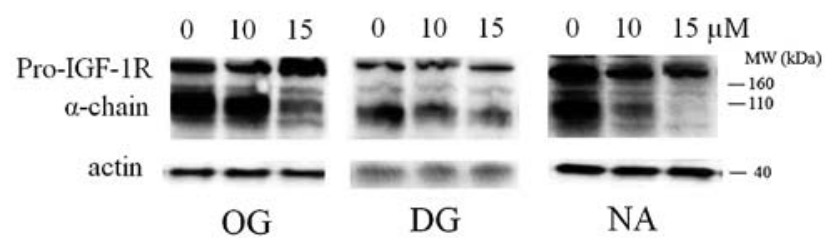

Figure 4. Effects of different concentrations of the polyphenols octyl gallate (OG), dodecyl gallate (DG) and nordihydroguariaretic acid (NA), as indicated in the figure, on the ratio between pro-IGF-1R and the corresponding mature processing product, the IGF-1R $\alpha$-chain, in HepG2 liver carcinoma cells. Expression levels of actin are shown as protein loading controls.

found by nordihydroguariaretic acid and octyl gallate while there was a moderate effect with dodecylgallate (Fig. 4).

Octyl gallate, dodecyl gallate and nordihydroguariaretic acid inhibit focus formation by HepG2 cells. Normal cells in culture stop growing when coming in physical contact with other cells or when a certain density is reached. Malignant cells, however, form areas of densely-packed cells piling up on the original monolayer of cells. This phenomenon is called focus formation. The relative number of foci formed is a measure for the degree of cell malignancy (19). For HepG2 cells the morphological changes that indicate a reversal of the malignant phenotype have been previously described $(20,21)$.

Cell viability/proliferation as a function of the concentration of the polyphenols was measured using the MTT assay. The relative viability/proliferation of cells grown during $48 \mathrm{~h}$ in culture medium containing increasing concentrations of the polyphenols is shown in Fig. 5A. Moderate concentrations ( $15 \mu \mathrm{M}$ and below) did only moderately compromise cell viability/proliferation in the HepG2 cell line during the $48 \mathrm{~h}$ incubation period. We subsequently tested octyl gallate, dodecyl gallate and nordihydroguariaretic acid for their capacity to reduce focus formation in HepG2 cells. Cells were grown in 6-well plates for 3 weeks and medium supplemented with different concentrations of octyl gallate, dodecyl gallate or nordihydroguariaretic acid was changed every other day. The results are shown in Fig. 5B. In the absence of the inhibitors, cells continue to grow and form foci. With increasing concentrations of the inhibitors, the number and size of the foci reduced indicating a gradual reversal of the malignant phenotype of the HepG2 cells (in each experiment around 100 foci were counted in the control).

Octyl gallate, dodecyl gallate and nordihydroguariaretic acid inhibit anchorage-independent growth of HepG2 cells. Despite the predictive value of the focus forming assay, anchorage-independent growth remains one of the hallmarks of transformation (22). We therefore also investigated the inhibition of anchorage-independent growth of HepG2 cells by octyl gallate, dodecyl gallate and nordihydroguariaretic acid. In the absence of the inhibitors, the liver carcinoma cell line readily formed multiple large colonies as illustrated in Fig. 5C. In the presence of different concentrations of the inhibitors, the colony size as well as the number of the colonies are significantly reduced (Fig. 5C), indicating that 
$\mathrm{A}$

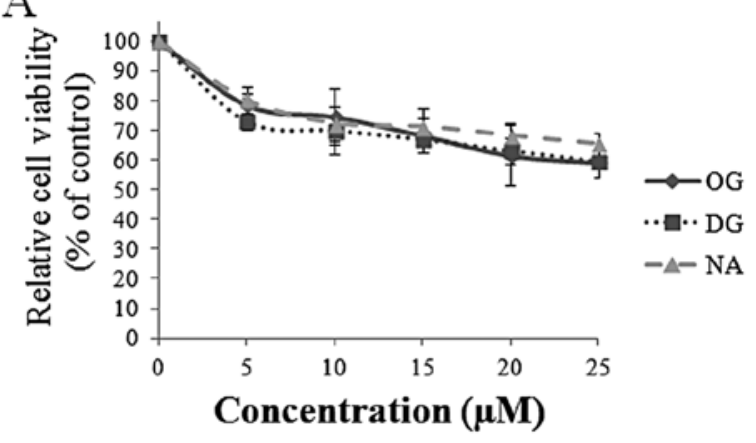

$\mathrm{B}$

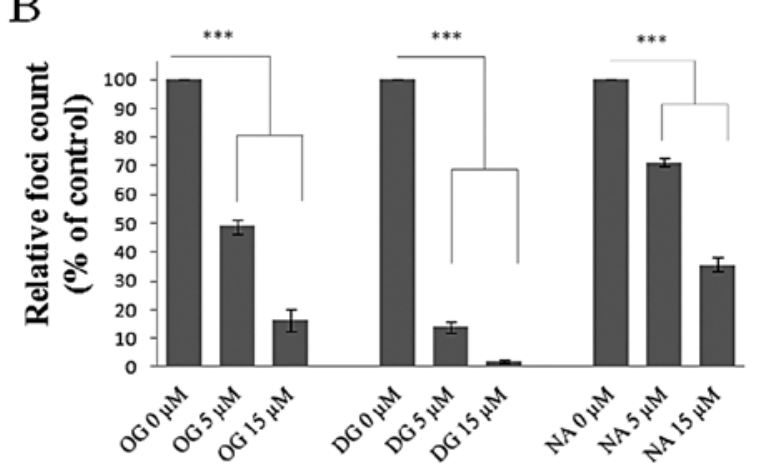

C

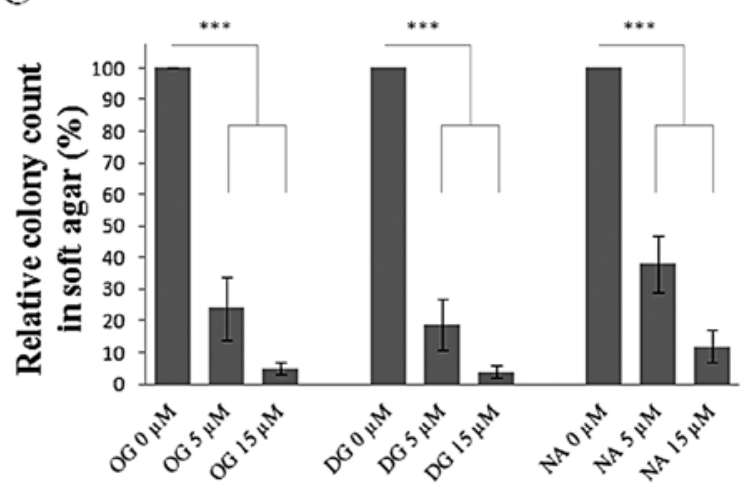

Figure 5. Impact of octyl gallate (OG), dodecyl gallate (DG) and nordihydroguariaretic acid (NA) on cell viability/proliferation, focus formation and anchorage-independent cell growth using HepG2 liver carcinoma cells (A) Graphs represent the relative cell viability/proliferation of the HepG2 cells in the presence of various concentrations of one of the three polyphenols. Data represent mean \pm SEM of 3 independent experiments, each performed in triplicate. (B) Impact of octyl gallate, dodecyl gallate and nordihydroguariaretic acid on focus formation using HepG2 liver carcinoma cells. Experiments were performed as described in Materials and methods. Foci counting results of treated cells are given relative to the results obtained with untreated cells. Data represent mean \pm SEM of 3 independent experiments, each performed in triplicate; $\left({ }^{* * *} \mathrm{p}<0.001\right.$ as compared to the untreated cells). (C) Impact of octyl gallate, dodecyl gallate and nordihydroguariaretic acid on anchorage-independent growth in soft agar using HepG2 liver carcinoma cells. Experiments were performed as described in Materials and methods. Colony counting results of treated cells are given relative to the results obtained with untreated cells. Data represent mean \pm SEM of 3 independent experiments, each performed in triplicate; $\left({ }^{* * *} \mathrm{p}<0.001\right.$ as compared to the untreated cells).

these inhibitors at the concentrations used effectively block a hallmark feature of tumor cells, i.e. anchorage-independent cell growth (in each experiment, around 50 colonies were counted in the control).

\section{Discussion}

In previous studies (3), PC inhibitory activities have been attributed to polyphenols and, subsequently, these were shown to be limited to inhibition only of the processing of a suitable fluorogenic peptide substrate in vitro (10). This limitation of inhibitory activities of polyphenols on PC-mediated proprotein processing could be explained, at least for a variety of polyphenols, to the ability of such compounds to bind to proteins as a result of the reactivity of their auto-oxidation products to proteins (11). The results of these studies indicate that polyphenols, in general, cannot be considered to constitute genuine PC inhibitors. Furthermore, these studies also reveal that the reported inhibitory effects of polyphenols on tumorigenic processes (23) are not mediated via direct interference of polyphenolic compounds with the functioning of PCs. In the present study, we investigated whether polyphenols could be affecting PCs via indirect mechanisms.

In an attempt to identify candidate polyphenols that could inhibit PCs in cells via indirect mechanisms, but not by direct interaction with the enzyme, different PC activity assays were performed. As expected on the basis of our previous studies $(10,11)$, many of the polyphenols inhibit the in vitro Pyr-RTKR-AMC peptide cleavage by hfurin ${ }^{573}$. On the other hand for most, if not all, of these polyphenols this inhibitory activity could not be demonstrated with the genuine protein PC substrate diphtheria toxin. The presence of protein could indeed protect the enzyme from auto-oxidation products of polyphenols (11).

In order to measure any possible indirect effect of polyphenols on PC activity (not dependent on direct interaction of the compound with the enzyme), $\mathrm{CHO}$ cells were incubated with a variety of polyphenols and, subsequently, the lysates of these cells were tested for the presence of PC activity by measuring Pyr-RTKR-AMC peptide cleavage. It is reasonable to assume that an observed reduction of PC activity after treatment of the cells with a particular polyphenol, as compared to the PC activity of control untreated cells, could be attributed to an effect resulting from the polyphenol treatment.

Using this approach, most of the tested polyphenols, which represented members of various classes of polyphenols, failed to reveal more than a modest impact on PC activity except for 5 different polyphenols, i.e. myricetin, octyl gallate, dodecyl gallate, ellagic acid and nordihydroguariaretic acid. Three polyphenols, i.e. octyl gallate, dodecyl gallate and nordihydroguariaretic acid were further studied. The results of these studies demonstrated that treatment of transfected $\mathrm{CHO}$ cells with these polyphenols revealed inhibition of processing of pro-IGF-1R and pro-GPC3 in a dose-dependent manner. This observation points strongly towards the inhibition of endogenous PC activity in the $\mathrm{CHO}$ cells by these different polyphenols.

Similar inhibitory activities by these polyphenols were observed in experiments in furin-deficient RPE.40 cells as well as in RPE. 40 cells in which both DNA encoding profurin and pro-IGF-1R were transfected. The observed impact of the polyphenols on the endogenous PC activity in $\mathrm{CHO}$ cells indicates that the inhibitory activity of these polyphenols is not specific for furin but most likely affects other PCs. If so, this 
would imply that these polyphenols affect a common property required for proprotein processing activity of the members of the PC family. In this context, it is noteworthy that all three polyphenols studied inhibited the maturation of pro-furin, which is mediated by an intramolecular autocatalytic process in the endoplasmic reticulum (24). With the exception of pro-PC2, PC maturation occurs in the ER (24-27). It is therefore possible that factors determining the local environment in the ER are influenced by the polyphenols which in turn influence PC maturation. Factors in the ER that could have impact on $\mathrm{PC}$ maturation include the $\mathrm{Ca}^{2+}$ concentration, the $\mathrm{pH}$ and the redox state.

Our results have shown that the inhibitory effect of octyl gallate, dodecyl gallate and nordihydroguariaretic acid on PC activity is associated with a reduction of both focus formation and anchorage-independent growth of HepG2 liver carcinoma cells. This is not surprising since inhibition of zymogen maturation and thus of PC activity have been shown to be sufficient to explain a reduction of anchorage-independent growth in HT-29 colon carcinoma cells (28). The importance of pro-IGF-1R processing was shown to be crucial in this context (28). The IGF axis is also increasingly recognized as one of the most important pathways in hepatocellular carcinoma (29). Activation of the IGF axis was observed in about 1 in 5 patents with early HCC (30). It has therefore been claimed that agents targeting this axis can potentially play an important role in the treatment or prevention of this type of cancer (29). Consequently, antiIGF-1R monoclonal antibodies were developed and tested in hepatocellular carcinoma cell lines (31). In this line of thinking, another study showed that overexpression of furin promoted HepG2 cell invasion in tail vein xenograft models (18). On the other hand, furin overexpression predicted a better postoperative disease-free survival in patients with hepatocellular carcinoma (32). Moreover, experiments with xenografts in which furin was overexpressed in Huh7 liver carcinoma cells demonstrated a significant suppression effect of tumor growth as compared to the mock control (32). Therefore it appears that influencing PC activity which affects many targets may exert different effects on liver tumor growth. The matter is further complicated by the finding that, at least in mice, organ microenvironment also affects growth and metastasis of hepatocellular carcinoma (33). The local levels of TGF- $\beta 1$, the pro-form of which is activated by PC activity, correlated with tumor metastasis (33). Our findings demonstrate that polyphenols might exert effects on liver tumorigenesis.

It should be noted that the polyphenols investigated in this study can affect tumor cells also via mechanisms other than an indirect inhibitory impact on proprotein convertase. It has been reported that octyl gallate and dodecyl gallate induce apoptotic cell death in cultured melanoma cells via alterations in apoptotic proteins expression. Both polyphenols were found to trigger cellular oxidative stress and to induce expression of proapoptotic Bax and to reduce expression of anti-apoptotic Bcl-2 (34). Furthermore, with other gallate derivatives apoptotic effects were observed with other tumor cells, such as various breast cancer cell lines (35), glioma cell lines (36) and lymphoma cell lines (37). This indicates an apoptotic effect of these polyphenols on a spectrum of tumor cells and, therefore, more wide-spread treatment possibilities.
With respect to nordihydroguariaretic acid, it has been demonstrated that it can restore tumor suppressor gene activity via demethylation of the tumor suppressor gene in human cancer cell lines (38), suggesting an impact on tumor cells via an epigenetic mechanism. Epigenetic alterations, heritable, but potentially reversible changes in DNA methylation and chromatin structure, constitute promising targets for cancer prevention strategies, since they occur early in the tumorigenic process, potentially even as initiating events for cancer development. The influence of natural product dietary components, including polyphenols, on the epigenome has received a major focus in recent epigenetic research. During the tumorigenic process a variety of cellular pathways can become deregulated by epigenetic alterations, such as regulation of the cell cycle, DNA repair mechanisms, apoptosis, cell growth control and differentiation. This occurs via epigenetic silencing of tumor suppressor genes, cell cycle regulators, apoptosis-inducing and DNA repair genes, and transcription factors by promoter methylation, as well as by modifications of histones and non-histone proteins by methylation or acetylation. Natural products, such as some polyphenolic compounds, can counteract these by influencing the activity or expression of DNA methyltransferases and histone modifying enzymes. In light of this, the use of plant polyphenolic compounds in chemoprevention is being considered.

In conclusion, our studies indicate that it is advisable to take the indirect proprotein convertase inhibitory activity of some plant polyphenols into account when natural products are considered for tumor treatment or for chemoprevention of carcinogenesis, including liver carcinogenesis $(39,40)$.

\section{Acknowledgements}

The authors acknowledge Dr. I. Lindberg for the soluble, recombinant human furin fragment (hfurin573) produced using a truncated human FUR cDNA construct (41). This hfurin573 was prepared for professor W. Van de Ven under Material Transfer Agreement of April 18, 2003, between Louisiana State University and KU Leuven R\&D and produced and purified as earlier described (42). This research was supported by 'Geconcerteerde Onderzoeksactie' (GOA-08/016), Project 324000 of K.U. Leuven Research \& Development, the 'Fonds voor Wetenschappelijk Onderzoek Vlaanderen' (FWO), the 'Industrieel Onderzoeksfonds' (IOF$\mathrm{HB} / 06 / 040)$ of KU Leuven and the Belgian Federation against Cancer.

\section{References}

1. Pratheeshkumar P, Sreekala C, Zhang Z, et al: Cancer prevention with promising natural products: mechanisms of action and molecular targets. Anticancer Agents Med Chem 12: 1159-1184, 2012

2. Nichenametla SN, Taruscio TG, Barney DL and Exon JH: A review of the effects and mechanisms of polyphenolics in cancer. Crit Rev Food Sci Nutr 46: 161-183, 2006.

3. Majumdar S, Mohanta BC, Chowdhury DR, Banik R, Dinda B and Basak A: Proprotein convertase inhibitory activities of flavonoids isolated from Oroxylum indicum. Curr Med Chem 17: 2049-2058, 2010.

4. Van de Ven WJ, Voorberg J, Fontijn R, et al: Furin is a subtilisin-like proprotein processing enzyme in higher eukaryotes. Mol Biol Rep 14: 265-275, 1990. 
5. Van de Ven WJ, Creemers JW and Roebroek AJ: Furin: the prototype mammalian subtilisin-like proprotein-processing enzyme. Endoproteolytic cleavage at paired basic residues of proproteins of the eukaryotic secretory pathway. Enzyme 45: 257-270, 1991

6. Khatib AM, Siegfried G, Chretien M, Metrakos P and Seidah NG: Proprotein convertases in tumor progression and malignancy: novel targets in cancer therapy. Am J Pathol 160 1921-1935, 2002.

7. Taylor NA, Van de Ven WJ and Creemers JW: Curbing activation: proprotein convertases in homeostasis and pathology. FASEB J 17: 1215-1227, 2003

8. Thomas G: Furin at the cutting edge: from protein traffic to embryogenesis and disease. Nat Rev Mol Cell Biol 3: 753-766, 2002 .

9. Chretien M, Seidah NG, Basak A and Mbikay M: Proprotein convertases as therapeutic targets. Expert Opin Ther Targets 12: $1289-1300,2008$

10. Zhu J, Declercq J, Creemers JWM, et al: Limitations of inhibitory activities of polyphenols on furin-mediated substrate processing. Curr Med Chem 19: 3641-3650, 2012.

11. Zhu J, Van de Ven WJ, Verbiest T, et al: Polyphenols can inhibit furin in vitro as a result of the reactivity of their auto-oxidation products to proteins. Curr Med Chem 20: 840-850, 2012.

12. Spence MJ, Sucic JF, Foley BT and Moehring TJ: Analysis of mutations in alleles of the fur gene from an endoproteasedeficient Chinese hamster ovary cell strain. Somat Cell Mol Genet 21: 1-18, 1995

13. Peretz S, Jensen R, Baserga R and Glazer PM: ATM-dependent expression of the insulin-like growth factor-I receptor in a pathway regulating radiation response. Proc Natl Acad Sci USA 98: 1676-1681, 2001.

14. Veugelers M, Cat BD, Muyldermans SY, et al: Mutational analysis of the GPC3/GPC4 glypican gene cluster on Xq26 in patients with Simpson-Golabi-Behmel syndrome: identification of loss-of-function mutations in the GPC3 gene. Hum Mol Genet 9: 1321-1328, 2000.

15. Van Duijnhoven HL, Creemers JW, Kranenborg MG, et al: Development and characterization of a panel of monoclonal antibodies against the novel subtilisin-like proprotein processing enzyme furin. Hybridoma 11: 71-86, 1992.

16. Mitchell DJ, Kim DT, Steinman L, Fathman CG and Rothbard JB: Polyarginine enters cells more efficiently than other polycationic homopolymers. J Pept Res 56: 318-325, 2000 .

17. Zhang YC, Wang XP, Zhang LY, Song AL, Kou ZM and Li XS Effect of blocking IGF-I receptor on growth of human hepatocellular carcinoma cells. World J Gastroenterol 12: 3977-3982, 2006.

18. Chen RN, Huang YH, Lin YC, et al: Thyroid hormone promotes cell invasion through activation of furin expression in human hepatoma cell lines. Endocrinology 149: 3817-3831, 2008.

19. Roy AM, Baliga MS and Katiyar SK: Epigallocatechin-3gallate induces apoptosis in estrogen receptor-negative human breast carcinoma cells via modulation in protein expression of p53 and Bax and caspase-3 activation. Mol Cancer Ther 4 : 81-90, 2005

20. Wilgenbus KK, Kirkpatrick CJ, Knuechel R, Willecke K and Traub O: Expression of Cx26, Cx32 and Cx43 gap junction proteins in normal and neoplastic human tissues. Int J Cancer 51: 522-529, 1992

21. Yano T, Hernandez-Blazquez FJ, Omori $\mathrm{Y}$ and Yamasaki $\mathrm{H}$ : Reduction of malignant phenotype of HEPG2 cell is associated with the expression of connexin 26 but not connexin 32 Carcinogenesis 22: 1593-1600, 2001.

22. Kang JS and Krauss RS: Ras induces anchorage-independent growth by subverting multiple adhesion-regulated cell cycle events. Mol Cell Biol 16: 3370-3380, 1996.

23. Yang CS, Wang H, Li GX, Yang Z, Guan F and Jin H: Cancer prevention by tea: evidence from laboratory studies. Pharmacol Res 64: 113-122, 2011.
24. Creemers JWM, Vey M, Schafer W, et al: Endoproteolytic cleavage of its propeptide is a prerequisite for efficient transport of furin out of the endoplasmic-reticulum. J Biol Chem 270: 2695-2702, 1995

25. Lindberg I: Evidence for cleavage of the Pc1/Pc3 pro-segment in the endoplasmic-reticulum. Mol Cell Neurosci 5: 263-268, 1994.

26. Muller L, Zhu X and Lindberg I: Mechanism of the facilitation of PC2 maturation by 7B2: involvement in ProPC2 transport and activation but not folding. J Cell Biol 139: 625-638, 1997.

27. Zhou A, Paquet L and Mains RE: Structural elements that direct specific processing of different mammalian subtilisinlike prohormone convertases. J Biol Chem 270: 21509-21516, 1995.

28. Khatib AM, Siegfried G, Prat A, et al: Inhibition of proprotein convertases is associated with loss of growth and tumorigenicity of HT-29 human colon carcinoma cells - importance of insulin-like growth factor-1 (IGF-1) receptor processing in IGF-1-mediated functions. J Biol Chem 276: 30686-30693, 2001.

29. Wu J and Zhu AX: Targeting insulin-like growth factor axis in hepatocellular carcinoma. J Hematol Oncol 4: 30, 2011.

30. Tovar V, Alsinet C, Villanueva A, et al: IGF activation in a molecular subclass of hepatocellular carcinoma and pre-clinical efficacy of IGF-1R blockage. J Hepatol 52: 550-559, 2010.

31. Yue L, Wang Y, Wang H, et al: Inhibition of hepatocellular carcinoma cell growth by an anti-insulin-like growth factor-I receptor monoclonal antibody. Oncol Rep 28: 1453-1460, 2012 .

32. Huang YH, Lin KH, Liao CH, Lai MW, Tseng YH and Yeh CT: Furin overexpression suppresses tumor growth and predicts a better postoperative disease-free survival in hepatocellular carcinoma. PLoS One 7: e40738, 2012.

33. Li G, Qin L, Ye Q, Dong Q, Ren N and Jia H: Organ microenvironment affects growth and metastasis of hepatocellular carcinoma via the TGF- $\beta /$ Smad pathway in mice. Exp Ther Med 5: 133-137, 2013.

34. De Cordova CA, Locatelli C, Assunção LS, et al: Octyl and dodecyl gallates induce oxidative stress and apoptosis in a melanoma cell line. Toxicol In Vitro 25: 2025-2034, 2011.

35. Calcabrini A, García-Martínez JM, González L, et al: Inhibition of proliferation and induction of apoptosis in human breast cancer cells by lauryl gallate. Carcinogenesis 27: 1699-1712, 2006.

36. Lee SH, Kim JK, Kim DW, et al: Antitumor activity of methyl gallate by inhibition of focal adhesion formation and Akt phosphorylation in glioma cells. Biochim Biophys Acta 1830: 4017-4029, 2013.

37. Serrano A, Palacios C, Roy G, Cespón C, Villar ML, Nocito M and González-Porqué P: Derivatives of gallic acid induce apoptosis in tumoral cell lines and inhibit lymphocyte proliferation. Arch Biochem Biophys 350: 49-54, 1998.

38. Cui Y, Lu C, Liu L, et al: Reactivation of methylation-silenced tumor suppressor gene p16INK4a by nordihydroguaiaretic acid and its implication in G1 cell cycle arrest. Life Sci 82: 247-255, 2008.

39. Stagos D, Amoutzias GD, Matakos A, Spyrou A, Tsatsakis AM and Kouretas D: Chemoprevention of liver cancer by plant polyphenols. Food Chem Toxicol 50: 2155-2170, 2012.

40. Darvesh AS and Bishayee A: Chemopreventive and therapeutic potential of tea polyphenols in hepatocellular cancer. Nutr Cancer 65: 329-344, 2013

41. Creemers JW, Usac EF, Bright NA, et al: Identification of a transferable sorting domain for the regulated pathway in the prohormone convertase PC2. J Biol Chem 271: 25284-25291, 1996.

42. Kacprzak MM, Peinado JR, Than ME, et al: Inhibition of furin by polyarginine-containing peptides: nanomolar inhibition by nona-D-arginine. J Biol Chem 279: 36788-36794, 2004. 